\title{
Application of Micro-single-hole Laparoscopy Combined with Hysteroscopy in Diagnosis and Treatment of Complex Uterine Cavity Diseases
}

\author{
Yilin Sun, Ying Cao, Jiming Chen*, Zhiyong Dong, Yafeng Zheng, Yunfen Jiang, Ruxia Shi \\ Department of Obstetrics and Gynecology, the Affiliated Changzhou No. 2 People's Hospital of Nanjing Medical University, Changzhou, \\ China
}

\author{
Email address: \\ cjming@126.com (Jiming Chen) \\ ${ }^{*}$ Corresponding author
}

\section{To cite this article:}

Yilin Sun, Ying Cao, Jiming Chen, Zhiyong Dong, Yafeng Zheng, Yunfen Jiang, Ruxia Shi. Application of Micro-single-hole Laparoscopy Combined with Hysteroscopy in Diagnosis and Treatment of Complex Uterine Cavity Diseases. Journal of Gynecology and Obstetrics. Vol. 9, No. 1, 2021, pp. 9-13. doi: 10.11648/j.jgo.20210901.13

Received: January 18, 2021; Accepted: February 6, 2021; Published: February 27, 2021

\begin{abstract}
Object: To investigate the feasibility and safety of hysteroscopic surgery for diagnosis and treatment of complex uterine cavity diseases under the monitor of $5 \mathrm{~mm}$-mini-incision micro-single-hole laparoscopic surgery. Methods: A retrospective analysis of 15 patients with complex uterine cavity diseases undergoing hysteroscopic surgery underwent 5mm-mini-incision micro-single-hole laparoscopic surgery was performed in the Department of Obstetrics and Gynecology, the Affiliated Changzhou NO. 2 People's Hospital of Nanjing Medical University from April 2018 to March 2019. Among them, 8 cases were intrauterine adhesion, 3 cases were uterine submucosal fibroid, 3 cases were uterine septum, 1 case was post-cesarean scar pregnancy, ages from 23 to 45 years old, BMI $17.65-25.48 \mathrm{~kg} / \mathrm{m}^{2}$. The operation was performed through a $5 \mathrm{~mm}$-mini-incision from the midline of the umbilicus. The 40/50 small abdominal retractor was used to expand the incision approach. A 3-mm small lens (A cystoscope or a hysteroscope was used for some patients) was placed into the single-port approach to perform the laparoscopic surgery; hysteroscopic surgery was performed by hysteroscopy through the cervix. Results: All the 15 patients were successfully operated. One patient with severe intrauterine adhesion had uterine perforation during operation. The uterus was repaired and sutured under micro-single-hole laparoscopy perfectly. No other channels were added during operation, and no laparotomy was performed. The operation time was 20-125 min, in which the surgical path establishment time was 5-10 min, the umbilical reconstruction time was 3-5 min; the intraoperative blood loss was 5-10 ml, and the postoperative hospital stay was 3-7 days. The postoperative umbilical incision healed well, and there was no surgical scar in the abdomen after surgery. No postoperative umbilical hernia and other serious complications occurred. Conclusion: Under the premise of mature umbilical single-port laparoscopy, it is safe and effective to use 5mm-mini-incision micro-single-hole laparoscopic surgery as a monitor during hysteroscopy for the diagnosis and treatment of complex uterine cavity diseases. It will achieve a better cosmetic result than traditional laparoscopy.
\end{abstract}

Keywords: 5mm-mini-incision, Micro-single-Hole Laparoscopy, Hysteroscopy, Complex Uterine Cavity Diseases

\section{Introductions}

Hysteroscopy is a good way to monitor the internal environment of the uterine cavity directly, target the resection or separation of lesions, as well as avoid the intimal damage caused by blind uterine operations, which has become a standard method for diagnosis and treatment of uterine cavity diseases [1]. However, complex uterine diseases such as cesarean scar pregnancy (CSP, endogenous type), uterine mediastinum, intrauterine adhesion, uterine submucosal fibroid, etc., have a greater risk of uterine perforation and adjacent organ damage during simple hysteroscopic surgery. Surgical procedures are often required under B-ultrasound or laparoscopic monitor [2]. With the continuous development of gynecological laparoscopic surgery, under the guidance of minimally invasive and "scar-free" concept, laparoendoscopic 
single-site surgery (LESS) has gradually become a new hotspot in minimally invasive surgery [3]. In addition to the advantages of reducing postoperative pain and promoting postoperative recovery, LESS also meets women's needs for beauty and brings patients more care. To minimize surgical incisions and surgical trauma is the goal of minimally invasive surgeons [4]. Based on the previous abundant experience of single-hole laparoscopic gynecological surgery, from April 2018 to March 2019, the endo-umbilical 5mm-mini-incision surgery had been successfully used to complete 15 cases of micro-single-hole laparoscopic hysteroscopic surgeries in the diagnosis and treatment of complex uterine diseases, and had achieved good results [5]. It is now reported.

\section{Materials and Methods}

\subsection{Clinical Data Basic Information}

A total of 15 patients who underwent hysteroscopic surgery under the endo-umbilical 5mm-mini-incision micro-single-hole laparoscopic surgery from April 2018 to March 2019 in our hospital were chosen in this study. Their ages range from 23-45 years, with a median age of 30, BMI range $17.65-25.48 \mathrm{~kg} / \mathrm{m}^{2} .8$ of the 15 patients had intrauterine adhesion, 3 uterine submucosal fibroid, 3 uterine septum, and 1 CSP (endogenous type).

\subsubsection{Case Selection Criteria}

Uniform body shape, and BMI $<30 \mathrm{~kg} / \mathrm{m}^{2}$; patient had stable vital signs and normal cardiopulmonary function, without contraindications for laparoscopic and hysteroscopic surgery, and with high risk factors for uterine perforation during simple hysteroscopic surgery and require laparoscopic surveillance.

\subsubsection{Case Exclusion Criteria}

Patients with abnormal spine and pelvis, who were unable to adopt bladder lithotripsy position; obesity patients with abdominal wall hypertrophy and puncture difficulty; patients with severe cardiopulmonary dysfunction or having taken long-term anticoagulant therapy; and patients with previous history of pelvic and abdominal surgery; patients with endometriosis and other serious pelvic adhesions; patients with a history of umbilical hernia; patients whose uterus was larger than 10 weeks of gestation; Other obvious contraindications for laparoscopic surgery were excluded.

\subsection{Surgical Methods}

\subsubsection{Preoperative Preparations}

Make conventional preoperative preparations, including exclusion of contraindications for laparoscopic surgery, routine umbilical cleansing before surgery, vaginal disinfection and scrubbing 1 day before surgery, fluid diet 2 to 3 days before surgery, intestinal preparation, placement of seaweed stick in the cervix to expand and soften the cervix at $10 \mathrm{pm}$ before surgery. Standard hysteroscopic instruments and special $3 \mathrm{~mm}$ miniature laparoscopic instruments are available. The patients were placed in bladder lithotomy position.

\subsubsection{Surgical Approach Platform}

A 40/50 small disposable abdominal retractor was used to connect 6.5 disposable surgical glove as an access platform during the surgical operation. Cutting the thumb, middle finger and little finger of the glove and inserting $5 \mathrm{~mm}$ disposable plastic puncture trocar and pediatric surgical micro-puncture trocar respectively to construct instrument working channels (Figures 1-3).

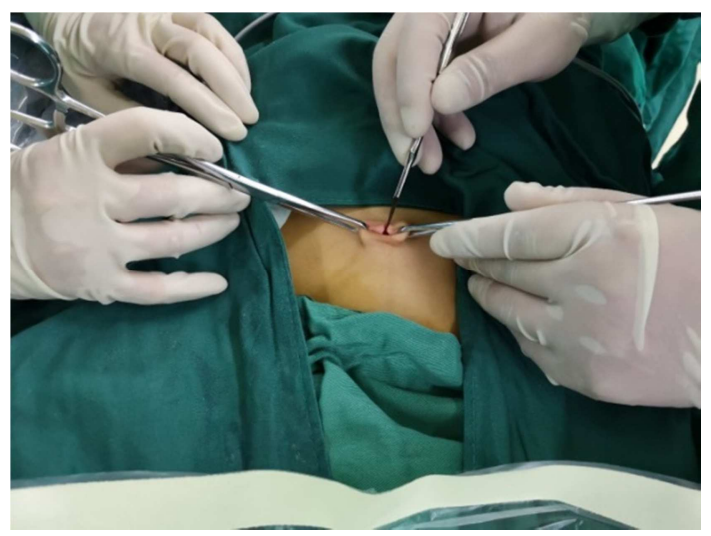

Figure 1. Surgical approach platform (1).

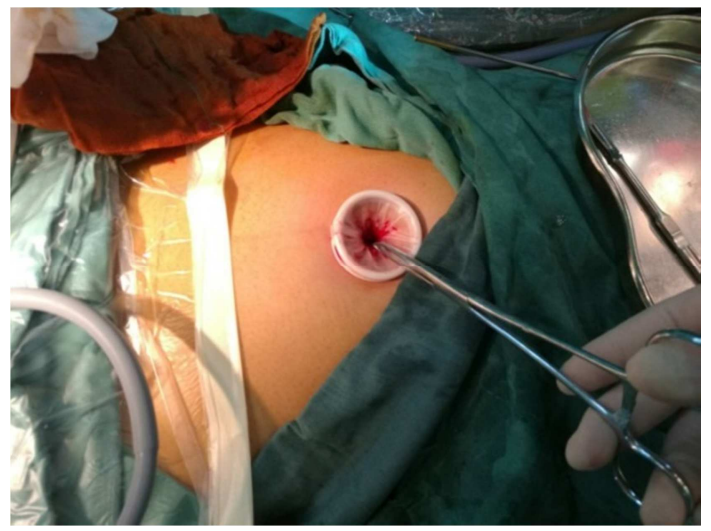

Figure 2. Surgical approach platform (2).

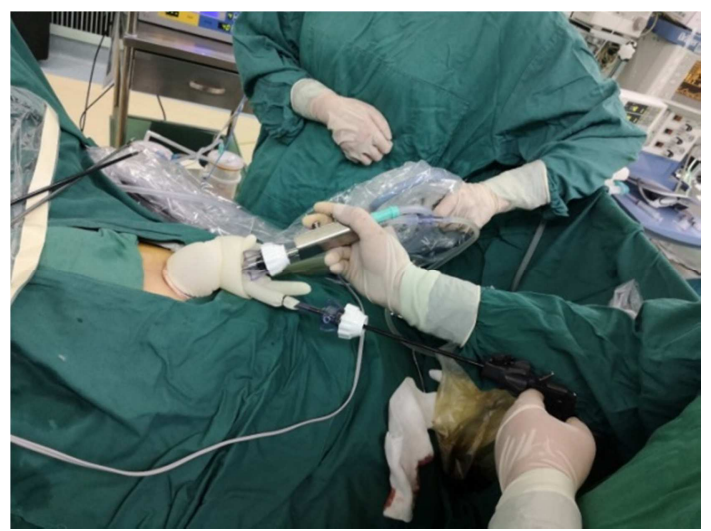

Figure 3. Surgical approach platform (3).

\subsubsection{Main Surgical Instruments}

The surgery was performed using a $3 \mathrm{~mm}$ small lens (One cystoscope or hysteroscopy was used for some patients.) and a dedicated $3 \mathrm{~mm}$ miniature laparoscopic instrument. Instruments mainly included Stryker digital laparoscopic 
system, 40/50 small disposable abdominal retractor, 2 pediatric surgical micro-puncture trocars, $15 \mathrm{~mm}$-trocar (in order to use ultrasonic knife), $30^{\circ}$ Storz $3 \mathrm{~mm}$-laparoscopic lens, light source, pneumoperitoneum system and 1 pair of $3 \mathrm{~mm}$-laparoscopic surgical scissors, separation forceps, ultrasonic scalpel, suction device, needle holder and bipolar electrocoagulation forceps respectively. Other special surgical consumables included 2-0 absorbed suture line (used for uterus suturing when uterine perforation occurs), and sodium hyaluronate (Figure 4).

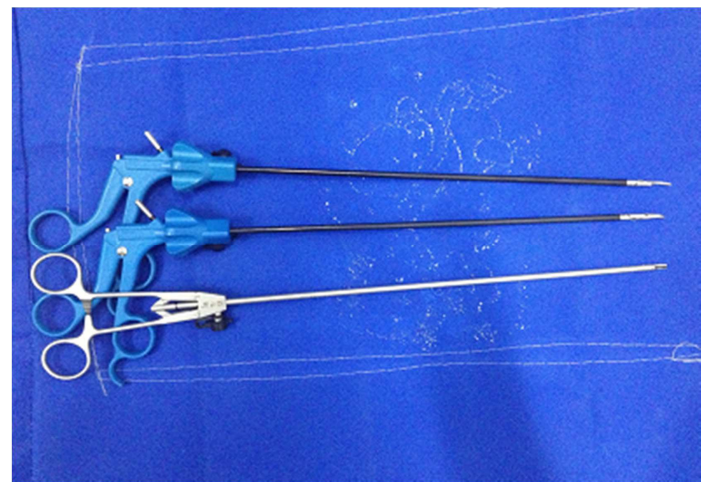

Figure 4. Main surgical instruments.

Establishment of surgical anesthesia, position and pathway

Patients underwent general anesthesia with endotracheal intubation and bladder lithotomy position. After routine disinfection and toweling, lift the umbilicus and take a longitudinal incision about $5 \mathrm{~mm}$ in the middle of the umbilicus, and the skin and subcutaneous tissue were cut into the peritoneum, and a 40/50 small disposable abdominal retractor was placed in the incision. Place the disposable glove on the incision retractor and fixing it with silk suture. Cut the small holes at the end of the finger of the glove and put the trocar into them $(5 \mathrm{~mm}$ disposable plastic puncture trocar was placed in the middle finger, pediatric surgical micro-puncture trocar was placed in the thumb and the little finger separately), filling with $\mathrm{CO}_{2}$ gas to form a satisfactory pneumoperitoneum, so that the intra-abdominal pressure was maintained between $10 \sim 12 \mathrm{mmHg}$. The pediatric surgical micro-puncture trocar with glove thumb was used to place the laparoscopic lens and connect the pneumoperitoneum machine; the other two trocars on the side were used to place the micro-operating forceps for surgical operation.

\section{Results}

Surgical conditions

All 15 patients were operated successfully. No other channels were added and none were converted to open procedures during the operation. One patient with severe intrauterine adhesion had uterine perforation during hysteroscopic surgery; under micro-single laparoscopic surgery, the perforation was repaired and sutured perfectly, and the patient recovered well. The adjacent organs such as the bladder and intestine were not damaged during the operation. The operation time was $20 \sim 125 \mathrm{~min}$, and the intraoperative blood loss was 5 10 $\mathrm{ml}$ (Tables 1-2).

Table 1. Clinical data of the patients $(n=15)$.

\begin{tabular}{|c|c|c|c|c|c|}
\hline Patient No. & Patient Age (years) & BMI $\left(\mathrm{kg} / \mathrm{m}^{2}\right)$ & Childbearing history & Pre-operative Diagnosis & Clinical manifestations \\
\hline 1 & 33 & 25.48 & G4P2 & uterine submucosal fibroid & menorrhagia, anemia \\
\hline 2 & 28 & 19.96 & G2P0 & intrauterine adhesion & scant menstrual \\
\hline 3 & 23 & 18.26 & G1P0 & intrauterine adhesion & amenorrhea \\
\hline 4 & 43 & 18.40 & G3P2 & post-cesarean scar pregnancy & -- \\
\hline 5 & 41 & 24.22 & G1P1 & uterine submucosal fibroid & menometrorrhagia, menorrhagia \\
\hline 6 & 39 & 19.81 & G4P1 & intrauterine adhesion & scant menstrual \\
\hline 7 & 30 & 21.51 & G2P1 & intrauterine adhesion & scant menstrual \\
\hline 8 & 26 & 24.97 & G1P0 & uterine septum & -- \\
\hline 9 & 28 & 21.05 & G1P1 & uterine septum & -- \\
\hline 10 & 34 & 17.65 & G5P1 & intrauterine adhesion & scant menstrual \\
\hline 11 & 28 & 21.50 & G5P0 & intrauterine adhesion & amenorrhea \\
\hline 12 & 27 & 22.22 & $\mathrm{G} 2 \mathrm{P} 0$ & uterine septum & -- \\
\hline 13 & 24 & 21.76 & G1P0 & intrauterine adhesion & amenorrhea \\
\hline 14 & 45 & 18.67 & G1P1 & uterine submucosal fibroid & menorrhagia, anemia \\
\hline 15 & 24 & 20.21 & $\mathrm{G} 2 \mathrm{P} 0$ & intrauterine adhesion & scant menstrual \\
\hline
\end{tabular}

Postoperative recovery

All patients had no obvious vaginal bleeding after operation. There was no need to use analgesic drugs after surgery routinely, and they could get out of bed within 24 hours. The patient was discharged from hospital after 3 to 7 days. No postoperative complications such as wound infection, incisional hernia, bladder dysfunction, subcutaneous emphysema and venous thrombosis occurred. The patients recovered well and were satisfied with the treatment. The patients are currently in follow-up observation.

Beauty Effect

The umbilical micro-single-hole laparoscopic surgery only takes a small incision of about 5-mm in the umbilical depression as the surgical approach. After the operation, the incision is sutured intradermally, and the umbilicus folds can conceal the incision well and no scar is left behind. The beauty effect is better than traditional laparoscopy (Figure 5). 
Table 2. Operative outcomes of the patients $(n=15)$.

\begin{tabular}{|c|c|c|c|c|}
\hline Patient No. & Operative time (min) & Blood loss (ml) & Hospitaliazation time after operation (d) & Complications \\
\hline 1 & 60 & 10 & 7 & -- \\
\hline 2 & 25 & 5 & 3 & -- \\
\hline 3 & 30 & 10 & 4 & -- \\
\hline 4 & 20 & 10 & 5 & -- \\
\hline 5 & 125 & 10 & 7 & -- \\
\hline 6 & 65 & 5 & 6 & -- \\
\hline 7 & 55 & 10 & 6 & -- \\
\hline 8 & 60 & 5 & 4 & -- \\
\hline 9 & 30 & 5 & 4 & -- \\
\hline 10 & 35 & 5 & 7 & uterine perforation \\
\hline 11 & 100 & 5 & 5 & -- \\
\hline 12 & 35 & 5 & 5 & -- \\
\hline 13 & 70 & 5 & 4 & -- \\
\hline 14 & 80 & 10 & 5 & -- \\
\hline 15 & 65 & 5 & 7 & -- \\
\hline
\end{tabular}

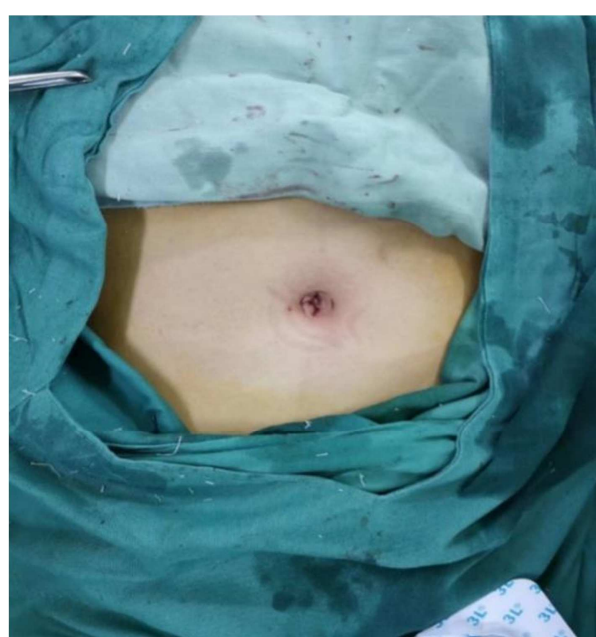

Figure 5. Beauty effect.

\section{Discussions}

Hysteroscopic surgery has become a standard method for diagnosis and treatment of uterine cavity diseases, it can remove the uterine cavity lesions, separate adhesions, correct the uterine cavity malformation, and restore the normal anatomy of the uterine cavity intuitively and accurately [6-8]. When uterine submucosal fibroids are large, especially type 2 submucosal fibroids, severe intrauterine adhesions, uterus septum, CSP (endogenous type), have high risk of uterine perforation and adjacent organs thermal damage while undergoing hysteroscopic surgery, and requiring B-ultrasound or laparoscopic monitoring [9]. On one hand, using B-ultrasound can reduce the occurrence of uterine perforation to a certain extent; on the other hand, B-ultrasound can only compare filling bladder with uterus expansion, guiding and monitoring surgical process indirectly. If the patient's abdominal fat is thick, it is difficult to locate hysteroscopic depth accurately; and ultrasound monitoring is largely limited by the level of the sonographer; once the uterine perforation or adjacent organ damage occurs, it is often necessary to operate laparoscopic surgery or open surgery. Compared with B-ultrasound, laparoscopic surgery can help to separate pelvic adhesions, drain the intestines away from the uterus, not only observing the internal environment of the abdominal cavity directly, but also performing hysteroscopic surgery; once perforation occurs, the uterus can be repaired immediately under laparoscopy to avoid excessive bleeding or severe adjacent organ damage. However, traditional laparoscopic surgery requires at least 3 puncture holes on the abdominal wall, the size ranges from 0.5 to $1.5 \mathrm{~cm}$. When the uterine perforation occurs, even 4 puncture holes are needed for uterine suturing. Although intestinal damage is avoided, the puncture scar is left inevitable. For female patients, especially young patients, there is still a problem of aesthetics deterioration. Minimizing surgical trauma is the goal of every surgeon and the direction of minimally invasive surgery. In view of this, LESS, with better cosmetic results, comes into being [10].

LESS, which produces a hole incision with a length of about $1.5 \sim 3.0 \mathrm{~cm}$ through the umbilicus has a better cosmetic effect compared with traditional laparoscopic surgery [11]. However, due to the destruction of the normal shape of the umbilicus, the scar will be left, more or less [12]. At the same time, the complete incision and suture of the normal structure of the umbilicus may increase the incidence of umbilical incisional hernia [13]. Hysteroscopic surgery under single-hole laparoscopy is the focus of the operation. The main function of laparoscopy is to monitor. Uterine perforation or diathermy damage rarely occurs. If the incision is further reduced to keep the umbilical pore morphological structure be not destroyed, on one hand, the cosmetic effect can be increased, on the other hand, the umbilical incisional hernia can be further reduced. Based on this concept and proficiency of traditional LESS, the author further reduced the umbilical incision and performed hysteroscopic surgery under the monitoring of 5mm-mini-incision LESS, not only increasing the safety of the operation, but also achieving better cosmetic results and higher patient satisfaction. All the 15 patients in this group were successfully operated, and achieved satisfied clinical results. One of the patients had uterine perforation during trans-cervical resection of uterus adhesion, and the perforation was successfully repaired under micro-single-hole laparoscopic surgery. No more severe 
consequences occurred. 5mm-mini-incision LESS surveillance hysteroscopic surgery with less pain, less time consuming of intestinal function recovery, has won high patient self-form satisfaction. Because LESS is a minimally invasive operation through a single site, there is a "chopstick effect". Compared with traditional endoscopic surgery, LESS is difficult to operate [14]. Once uterine perforation or diathermy damage occurs, the uterus or intestine should be sutured immediately, but due to the small operation space of $5 \mathrm{~mm}$-mini-incision, the operation time must be further extended and 5mm-mini-incision LESS requires special surgical instrument and multi-channel puncture cannula, it is time-consuming to establish channel. LESS is highly dependent on equipment and operating skills. Therefore, the implementation of $5 \mathrm{~mm}$-mini-incision LESS has higher requirements. It is better to be prepared fully and well-trained to reduce the risk of surgery effectively and ensure the success of the operation [15].

\section{Conclusions}

In conclusion, this study initially confirmed that 5mm-mini-incision micro-single-hole laparoscopic surveillance for hysteroscopic surgery is safe and feasible for the diagnosis and treatment of complex uterine cavity diseases, even uterine perforation or peripheral organ diathermy damage occurs, the $5 \mathrm{~mm}$-mini-incision LESS can suture and repair immediately; however, the $5 \mathrm{~mm}$-mini-incision LESS may require longer operation time and is more difficult to perform, relying on experienced surgeons. The safety and efficacy of hysteroscopic surgery under the monitoring of $5 \mathrm{~mm}$-mini-incision LESS will be further confirmed by prospective, randomized large-sample studies.

\section{Funding}

This work was supported by grants from the Scientific Research Support Program for Postdoctoral of Jiangsu Province (2019K064), the Major Science and Technology Program of Changzhou Health and Family Planning Commission (ZD201812), the Scientific Research Support Program for "333 Project" of Jiangsu Province (BRA2019161).

\section{References}

[1] Centini G, Troia L, Lazzeri L, Petraglia F, Luisi S. Modern operative hysteroscopy. Minerva Ginecol. 2016; 68 (2): 126-132.
[2] Vigoureux S, Fernandez H, Capmas P, Levaillant JM, Legendre G. Assessment of Abdominal Ultrasound Guidance in Hysteroscopic Metroplasty. Journal of minimally invasive gynecology. 2016; 23 (1): 78-83.

[3] Bradford LS, Boruta DM. Laparoendoscopic single-site surgery in gynecology: a review of the literature, tools, and techniques. Obstet Gynecol Surv. 2013; 68 (4): 295-304.

[4] Matanes E, Lauterbach R, Boulus S, Amit A, Lowenstein L. Robotic laparoendoscopic single-site surgery in gynecology: A systematic review. Eur J Obstet Gynecol Reprod Biol. 2018; 231: $1-7$.

[5] Yen CF, Chou HH, Wu HM, Lee CL, Chang TC. Effectiveness and appropriateness in the application of office hysteroscopy. $\mathrm{J}$ Formos Med Assoc. 2019; 118 (11): 1480-1487.

[6] Deffieux X, Gauthier T, Menager N, et al. Hysteroscopy: guidelines for clinical practice from the French College of Gynaecologists and Obstetricians. Eur J Obstet Gynecol Reprod Biol. 2014; 178: 114-122.

[7] Hoshino T, Yanagawa M, Matsubayashi AU, Yoshioka S. Useful technique for submucous myomectomy under direct transcervical resectoscope observation. Gynecol Minim Invasive Ther. 2017; 6 (3): 120-122.

[8] Xia EL, Duan H, Zhang J, et al. Zhonghua Fu Chan Ke Za Zhi. 2003; 38 (5): 280-283.

[9] Chen J, Lin X, Gao H, Ding Y, Deng Y, Yuan X, et al. Application of laparoendoscopic single-site surgery using conventional laparoscopic instruments in gynecological diseases. International Journal of Clinical and Experimental Medicine. 2016; 9 (7): 13099-104.

[10] Fader AN, Rojas-Espaillat L, Ibeanu O, Grumbine FC, Escobar PF. Laparoendoscopic single-site surgery (LESS) in gynecology: a multi-institutional evaluation. American Journal of Obstetrics and Gynecology. 2010; 203 (5): 501e1-e6.

[11] Ichikawa M, Akira S, Mine K, Ohuchi N, Iwasaki N, Kurose K, et al. Evaluation of Laparoendoscopic Single-site Gynecologic Surgery with a Multitrocar Access System. Journal of Nippon Medical School. 2011; 78 (4): 235-40.

[12] Ki EY, Park EK, Jeong IC, et al. Laparoendoscopic Single Site Surgery for the Treatment of Huge Ovarian Cysts Using an Angiocatheter Needle. Yonsei Med J. 2019; 60 (9): 864-869.

[13] Gunderson CC, Knight J, Ybanez-Morano J, Ritter C, Escobar $\mathrm{PF}$, Ibeanu $\mathrm{O}$, et al. The Risk of Umbilical Hernia and Other Complications with Laparoendoscopic Single-Site Surgery. Journal of minimally invasive gynecology. 2012; 19 (1): 40-5.

[14] Boruta DM. Laparoendoscopic single-site surgery in gynecologic oncology: An update. Gynecol Oncol. 2016; 141 (3): 616-623.

[15] Koo YJ. Recent advances in minimally invasive surgery for gynecologic indications. Yeungnam Univ J Med. 2018; 35 (2): $150-155$. 